\title{
Work in progress: a proposal for a hotspot metric to extend the lifetime in wireless sensor network
}

\begin{abstract}
Recently, the revolution of deploying wireless sensor network (WSN) technology has accelerated the need for further development. Thus, numerous researches of different perspectives seeking some enhancements have been proposed. Throughout our extensive study, we have found that there is a phenomenon with adverse side effects known as sink isolation (sink's hotspot zone) which exists due to the sink's neighbor nodes (deputy nodes) run out of energy faster than the others. This draws attention to our hypothesis, energy exhaustion in sink's hotspot zones is worthy of concern more than that of distant zones. Accordingly, we propose a pioneering metric with highly influential factors for forward node selection. This proper selection ends the route of the data traffic in a deputy node of least energy exhaustion. As a result, extensively dissipated sensors in the hotspot zones are avoided, sinks are protected from isolation, and lifetime is extended.
\end{abstract}

Keyword: WSN; Lifetime; Hotspot; Tree topology 